\title{
The temporal structure of spoken sentence comprehension in Chinese
}

\author{
PING LI \\ Chinese University of Hong Kong, Shatin, Hong Kong
}

\begin{abstract}
Three experiments with 60 native Chinese speakers were conducted to examine the temporal structure of spoken sentence comprehension in Chinese. In the two sentence-gating experiments, listeners heard sentence fragments of various sizes, either successively or individually, and decided on the agent role of the sentence. In the agent-naming experiment, listeners repeated aloud the sentence agent upon hearing the sentence. Converging evidence was derived from these experiments indicating the usefulness of sentence gating in tapping into the temporal structure of comprehension and in evaluating listeners' comprehension on the basis of partial information. Results from these experiments show that partial sentence information plays a very important role in Chinese sentence comprehension, and that Chinese listeners integrate incomplete and ungrammatical sentence fragments by reference to complete and grammatical models in the language. It is argued that these processing characteristics reflect properties of the Chinese language in which few morphological, grammatical, and syntactic constraints are placed on the relationships between sentence constituents.
\end{abstract}

Spoken sentence comprehension is a process by which the listener continuously evaluates various sources of information as the input signal accumulates over time. Just how the processor performs such evaluations and at which point in time it arrives at a correct interpretation is so far largely unclear. In spoken word recognition much work has examined the temporal aspects of processing. For example, research has shown that listeners can recognize a word in normal context with about only half of the acousticphonetic information of that word (Grosjean, 1980; MarslenWilson, 1987), and that the time course of lexical activation is traceable at different levels (Marslen-Wilson, 1990). Unfortunately, little work has tackled similar problems in the context of spoken sentence comprehension. This research is an attempt to provide some empirical evidence on the temporal structure of spoken sentence comprehension in Chinese, a language that has no inflectional morphology but that permits a larger range of sentence types than are possible in most Indo-European languages.

It is important to know not only whether listeners can interpret a sentence before having the complete acoustic

This research was supported by an Incentive Research Fund and a Direct Research Grant from the Chinese University of Hong Kong to P.L. and in part by a NIH/NIDCD Grant (RO1-DC00216-10) to E. Bates for the project "The Crosslinguistic Studies of Aphasia." I am deeply indebted to $\mathrm{E}$. Bates for her constructive and insightful discussions during various phases of this work. I would like to thank F. Grosjean, M. Bond, H. H. Pan, and H. Cheung for helpful comments on various versions of this paper. I would also like to thank L. Y. Tan, L. P. Yang, W. Wong, and R. Buffington for their assistance in the experiments. Correspondence concerning this article should be addressed to P. Li, Department of Psychology, The Chinese University of Hong Kong, Shatin, N.T., Hong Kong (e-mail: ping@psy.cuhk.hk). (Following the summer of 1996, P. L. will be at the Department of Psychology, University of Richmond, Richmond, VA 23173; e-mail: pingli@urvax.urich.edu) signal, but also when and how they do so. The issue of when has to do with the temporal structure, specifically, with which point in time a decision is made about sentence roles and how the decision changes along the course of the sentence. The issue of how has to do with the factors that act and interact to shape sentence comprehension processes and to determine decision speed at various time points.

In a recent study, P. Li, Bates, and MacWhinney (1993) examined some important factors in real-time spoken sentence comprehension in Chinese. A reaction time technique was used to study main effects and interactions among word order, noun animacy, the object marker $b a$, the passive marker $b e i$, and the indefinite marker $y i$. In the experiment listeners heard a sentence and simultaneously saw a pair of pictures that corresponded to the nouns in the sentence. Their task was to decide which noun represented the agent of the sentence, as quickly and as accurately as they could. Reaction time data as well as choice response data were collected and analyzed for the various factors or cues - a technical term in the Competition Model of Bates and MacWhinney (1982; MacWhinney \& Bates, 1989) referring to information sources that mediate the form-meaning mapping.

Results from the experiments indicated that Chinese speakers rely heavily on semantic cues such as noun animacy (e.g., animacy dominates word order cues in competition), consistent with previous untimed studies of sentence comprehension in Chinese (e.g., Liu, Bates, \& Li, 1992b; Miao, 1981; Miao, Chen, \& Ying, 1986). Chinese speakers also make important use of the markers $b a$ and $b e i$ in the interpretation of sentence roles. In Chinese, word order effect appears to vary with context unlike in English. The results also suggest that cues do not necessarily fall into clusters of linguistic types within the hierarchy of cue importance to sentence meaning. The data are compatible 
with interactive activation models of sentence comprehension, and at the same time pose problems for models that assume a modular architecture in which morphological, syntactic, and semantic sources of information are insulated from one another.

The reaction time data in P. Li et al. (1993) attest to the utility of real-time studies at the sentence level; they can reveal aspects of processing that are often either not available or obscure in studies using only decision measuresmeasures that have been relied on in most experiments involving agent role assignment (see MacWhinney \& Bates, 1989). However, the reaction-time method adopted in $\mathrm{P}$. Li et al. was still confined to "one decision only" for a given sentence, a method that does not reflect properties of spoken sentence comprehension as the sentence unfolds in time. So far, previous studies have provided evidence concerning only the role of different cues in sentence comprehension in Chinese. It is not yet clear how these cues interact at different temporal locations of the sentence. In order to evaluate comprehension at separate points of the sentence, the present research adapted the gating technique from spoken word recognition by asking listeners to identify the sentence agent on the basis of sentence fragments as well as full sentences. In particular, I ask the following questions: When does the listener decide on an interpretation of sentence role for a particular type of sentence? How does the interpretation change along the course of the sentence? What determines the speed at which the listener assigns a sentence role? And, how do cues interact to jointly contribute to the final interpretation of the sentence?

Three experiments were designed to examine these questions. The first two were sentence-gating experiments, adapted from the word-gating paradigm (Grosjean, 1980). Sentence materials were presented either successively (Experiment 1 ; see also P. Li, 1994) or individually (Experiment 2 ). The third was an agent-naming experiment, adapted from the word-shadowing paradigm (Liu, Bates, Powell, \& Wulfeck, in press; Marslen-Wilson, 1985; Slowiaczek, 1994). Consistent results from these different paradigms, I reasoned, will lead to converging evidence, while inconsistent results may reflect effects specific to a particular paradigm.

Before presenting the current research in detail, I will discuss some properties of Chinese that are particularly important for understanding sentence comprehension processes in this language (see P. Li et al., 1993, for a more detailed discussion of these properties; see also Aaronson \& Ferres, 1986, for relevant discussion).

First, Chinese grammar is characterized by its lack of inflectional morphology. There are no case markings, no tense suffixes, and no subject-verb agreement in terms of number or gender. Chinese linguists argue that, because the grammatical relations between major sentence constituents are not linked by morphological devices, there is no simple correspondence between grammatical roles of a sentence and the parts of speech of a word (Zhu, 1985). Related to this property is the high degree of ellipsis for major sentence constituents. Chinese is a "pro-drop" or null- subject language, in which a previously identified subject can often be omitted (Huang, 1984). In addition, Chinese allows null objects (Cole, 1987). Thus a simple Chinese sentence, when literally translated, would sound telegraphic in a richly inflected language.

Second, in addition to the canonical SVO sentences, Chinese permits several pragmatically conditioned word order variations that are illegal in English, including SOV, OSV, and VOS (C. Li \& Thompson, 1981; Lu, 1980). ${ }^{1}$ Considering the properties of ellipsis and word order variations together, one can anticipate that Chinese offers sentence status to many constructions that would be considered incomplete and/or ungrammatical in English (e.g., V, $\mathrm{OV}, \mathrm{VO}$ ). These facts may complicate the interpretation of a sentence-level study, but they also open up interesting questions about the role of partial information in spoken sentence comprehension, questions that could not be studied in English.

Third, Chinese has two important particles for the identification of sentence roles, the object marker $b a$ and the passive marker bei (Chao, 1968; C. Li \& Thompson, 1981; Sun, 1991). $B a$ marks the immediately following noun as the sentence object (usually the patient), and bei marks the same noun as the sentence agent. $B a$ and bei typically occur in NNV sentences, with Nba NV interpreted as SOV and $\mathrm{Nbe} i \mathrm{NV}$ as OSV. Because the noun following the bei marker can be legally omitted, the Chinese NbeiV sentence has the same functional role as the English passive with the truncated $b y$ phrase. NNV strings without $b a$ and bei are pragmatically conditioned: They can be used either to topicalize the sentence object (corresponding to an OSV interpretation) or to describe a situation in which the speaker provides information counter to the expectation of the listener (corresponding to an SOV interpretation).

Several features of $b a$ and $b e i$ should be noted here (see P. Li et al., 1993, for more details): (1) The verb following both the $b a$ and bei phrases must be structurally complex (i.e., single monosyllabic verbs cannot occur after $b a$ or bei); (2) ba but not bei requires that the following noun phrase be definite or specific; (3) the class of nouns that can occur after $b a$ may not always be the sentence patient, but the class of nouns following bei always indicates the sentence agent; and (4) ba but not bei is partially homophonous with sentence-final particles (although the sentencefinal particle $b a$ carries a neutral tone while the object marker $b a$ has a dipping tone). The last three points suggest that there exist some differences between $b a$ and $b e i$ in terms of their functional clarity in marking sentence roles.

These language-specific properties may have significant implications for spoken sentence comprehension in Chinese. First, given that many daily sentences consist of fragments with omissions, we may expect that partial information plays a special role in Chinese listeners' interpretation of sentence constituents. Second, because grammatical functions in Chinese are not overtly indicated by morphological or syntactic devices as they are in English or other Western languages, we may expect that the interpretation of sentence roles in Chinese will remain stable whether the sentence is grammatical or contains gramma- 
tical violations, unlike that in English (there is evidence that grammaticality plays an important role in sentence comprehension in English; see Bates, 1991; von Berger, Wulfeck, Bates, \& Fink, in press). Third, because of the importance of the $b a$ and bei markers, we may expect that their occurrence in the sentence will help Chinese listeners to assign sentence roles. However, the differences between $b a$ and bei with respect to their functional clarity might affect the weight with which they influence sentence role assignment (as found in P. Li et al., 1993). In the following, we shall see if there is empirical evidence for any of these expectations.

\section{EXPERIMENT 1}

Experiment 1 employed a sentence-gating task, a sentence-level adaptation of the gating method pioneered by Grosjean (1980) for the study of spoken word recognition. In the past decade the gating paradigm has been applied successfully to the study of monolingual and bilingual spoken word recognition (see Grosjean, 1980, 1988; Grosjean, Dommergues, Cornu, Guillelmon, \& Besson, 1994; Marslen-Wilson, 1987; Tyler \& Wessels, 1985). Evidence has accumulated that gating is particularly useful in examining the moment-to-moment recognition processes and in assessing the amount of phonetic-acoustic information needed for the correct identification of the word. Recently, we applied the gating method to a grammaticalityjudgment task with bilingual English-Chinese speakers (Liu, Bates, \& Li, 1992a), and the results proved encouraging: The gating method could be used to identify the temporal structure within which listeners make a grammaticality judgment in bilingual processing.

In the word-gating task, listeners are presented with fragments of a word, one at a time in increasing duration, until the whole word has been presented. The first fragment or gate starts from the beginning of the word with a duration of about $30-50 \mathrm{msec}$, and each remaining gate increases by about $30-50 \mathrm{msec}$ to the previous gate; this continues until the last gate when the whole word is presented. At each presentation, listeners are required to identify the word being presented on the basis of the partial information provided for that word (except the last gate, which contains the complete information of the word).

In the current adaptation of the word-gating paradigm, sentences are similarly gated into small fragments, but on a word-by-word basis. Listeners hear increasingly longer fragments of a sentence each time until the whole sentence has been presented. At each presentation, they are asked to assign the agent role to a sentence constituent, that is, to choose a noun that represents the sentence agent. The agent-choice task requires the listener to understand the relationships between the different sentence constituents and thus provides a good measure of sentence comprehension.

\section{Method}

\section{Participants}

Twenty native adult Mandarin Chinese speakers from mainland China participated in this experiment ( 11 females and 9 males, mean age $=29$ ). They were students or visiting scholars at the Chinese
University of Hong Kong. Most participants were from the northern areas in China (i.e., the Mandarin areas), and had stayed in Hong Kong for less than a year by the time of the experiment. All of them use Mandarin Chinese intensively in daily life, and only one could speak Cantonese.

\section{Materials}

Three sets of test sentences were used in this study: (1) 36 simple sentences with no markers, 12 each in the orders NVN, NNV, and VNN; (2) 36 sentences with the object marker $b a, 12$ each in the orders NVN, NNV, and VNN (with $b a$ occurring at three locations: before the first word, the second word, or the third word); and (3) 36 sentences with the passive marker bei, 12 each in the order NVN, NNV, and VNN (with bei occurring at three locations: before the first word, the second word, or the third word). The animacy cue was not varied in this experiment because the relative importance of animacy versus word order and the markers is clear from previous research. Nor was the indefinite marker included because it is a weak cue, as shown in previous experiments (P. Li et al., 1993). The selection of materials reflected a compromise between having the most important factors and limiting the possible number of factorial permutations; it also reflected a particular concern with structural information of the sentence (i.e., global syntactic information such as word order cues and local grammatical information such as the markers $b a$ and $b e i$ ).

The design included a within-subject variable, order (NVN, NNV, and VNN in all three sets) and a within-subject variable, gate size ( 1 , 2 , and 3 for simple sentences; $1,2,3$, and 4 for $b a$ and $b e i$ sentences). In addition, the $b a$ and $b e i$ sentences included a within-subject variable, position, a three-level variable reflecting the position of the $b a$ or bei marker within the sentence frame. Thus, the design for simple sentences was $3 \times 3$, while that for $b a$ and bei was $3 \times 4 \times 3$, respectively. This yielded a total of 81 test conditions.

Each test sentence contained two nouns and a verb. Appendix A lists all the nouns and verbs used in the experiment. All nouns and verbs were disyllabic since $b a$ and $b e i$ sentences typically require complex verbs (the verbs used in this study are resultative verb compounds, with the second component marking the result). Our computer program generated random combinations of the nouns and verbs for the 108 test sentences ( 36 each for simple, $b a$, and bei). Appendix $B$ lists in order all the test sentence types used in the experiment, together with judgments of their grammaticality. ${ }^{2}$ Note that the sentence gates included a mixture of grammatical and ungrammatical sets because of the full crossing of variables. The information in Appendix B shows that grammaticality "comes and goes" as fragments are added to form a complete sentence. In other words, sentences do not start out fine and then become irrevocably and monotonically bad, or vice versa. Rather, the grammaticality of a sentence gate depends on the information that will follow as well as the information that has already occurred (our study with English-Chinese bilingual speakers provided empirical evidence for the effect of "come-andgo" in grammaticality-judgment tasks; see Liu et al., 1992a).

\section{Experimental Apparatus}

All test sentences were recorded on a digital audiotape by a female native Mandarin Chinese speaker who was unaware of the experimental purpose. The sentences were read at a normal rate with a smooth intonation. They were then digitized into a Macintosh II computer through the analog-to-digital function of the AudioMedia device. During playback, the digital-to-analog function of AudioMedia converted the digital signal and sent the sound to a pair of amplified speakers. A sampling rate of $22 \mathrm{kHz}$ was used for digitizing and gating. In most cases it was not a problem to determine the word boundary and gate the sentence on a word-by-word basis by inspecting the speech wave forms. In a few cases where this was a problem, the onset of the gate was located as accurately as possible by enlarging the windows of the speech wave forms and by using auditory feedback repeatedly. 
Pictures were digitized with an Apple scanner, and during the experiment they were displayed on a high-resolution monitor in a $7 \times$ $11 \mathrm{~cm}$ frame.

The experiment was run on a Macintosh II. The presentation of auditory and pictorial materials was controlled by the PsyScope program (Cohen, MacWhinney, Flatt, \& Provost, 1993). The CMU button box, which was interfaced with PsyScope, was used to register participants' choice decisions and decision times.

\section{Procedure}

Before the experiment began, the experimenter explained the task in Mandarin Chinese to the listener. During the experiment, listeners heard the individual gates of a sentence successively in a sequential order. Each time they heard a sentence gate, they simultaneously saw on the computer screen a pair of pictures that corresponded to the two nouns described in the sentence. Their task was to determine, as quickly and as accurately as possible, which of the two pictures (appearing on the left or the right side of the screen, counterbalanced) represented the sentence agent. They were asked to indicate this choice by pressing the left or the right button on the button box. The onset of the sentence gate started the button box timer for the listeners' response times to that gate. Listeners were given $2 \mathrm{sec}$ after the gate offset to respond, before hearing the stimulus on the next trial. This amount of time was sufficient to allow full response for most listeners under most conditions (see below for miss rate), while putting time pressure on the response speed.

The experiment consisted of four blocks of testing ( 99 sentence gates in each). Each block contained a randomized combination of all sentence types. Participants completed the four blocks in two sessions, with two blocks in each session. There was a 5-min break after each block, and a 1-week interval between the two sessions. Each session lasted about $30 \mathrm{~min}$.

\section{Data Analysis}

Two dependent variables were measured in this experiment. The first was the listeners' choices of the sentence agent. Each listener's choice was given a score of 1 if the listener chose the first noun and 0 if he/she chose the second noun. A summary score was calculated for the four items within each cell, and the numbers were then entered into statistical analyses. Note that the percentage of the firstnoun choice was inversely related to the percentage of the secondnoun choice. Thus, a score close to $100 \%$ reflected that the first noun was reliably chosen as agent, while a score close to $0 \%$ re- flected that the second noun was reliably chosen. Missing responses were scored as 0.5 . The overall rate of missing values for this experiment was $3 \%$.

The second dependent variable was listeners' reaction times (RTs) to each of the sentence gates. The computer program calculated RTs from the beginning of the gate when it was played on the speaker to the time when listeners pushed the button. When a particular response was missing for a given trial, that trial was assigned the average RT of the particular listener in the final analysis.

\section{Results and Discussion}

In what follows, I will start by presenting results for the $3 \times 3$ (word order $\times$ gate size) analysis of the simple sentence types, looking first at choices and then at RTs. I will then present results for the analyses of the $b a$ and bei sentences, in the same order with choices being followed by RTs.

\section{Simple Sentences}

Choice data. Figure 1a presents the choice data for the simple sentences. Analysis of variance (ANOVA) on this set of data revealed that all three effects (i.e., the main effects of word order and gate size and the two-way interaction effect) were statistically significant. The main effect of gate size $[F(2,38)=28.78, p<.001]$ showed that listeners tended to change their interpretations across different locations of the sentence. The main effect of word order $[F(2,38)=85.75, p<.001]$ indicated that different orders led to different interpretations, in line with results from other studies using full sentence materials (P. Li et al., 1993; Miao, 1981; Miao et al., 1986). Once all the information was received (i.e., on the final gate), listeners chose the first noun as the sentence agent for NVN ( $93 \%$ first-noun choice), but chose the second noun for VNN (14\% firstnoun choice, i.e., $86 \%$ second-noun choice). However, listeners showed no preferences for either noun for NNV ( $54 \%$ first-noun choice at the final gate). This chancelevel performance for the complete NNV sentences clearly

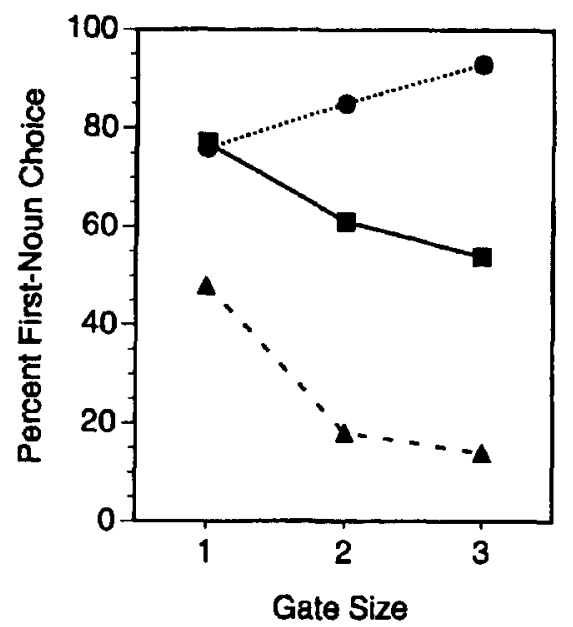

(a)

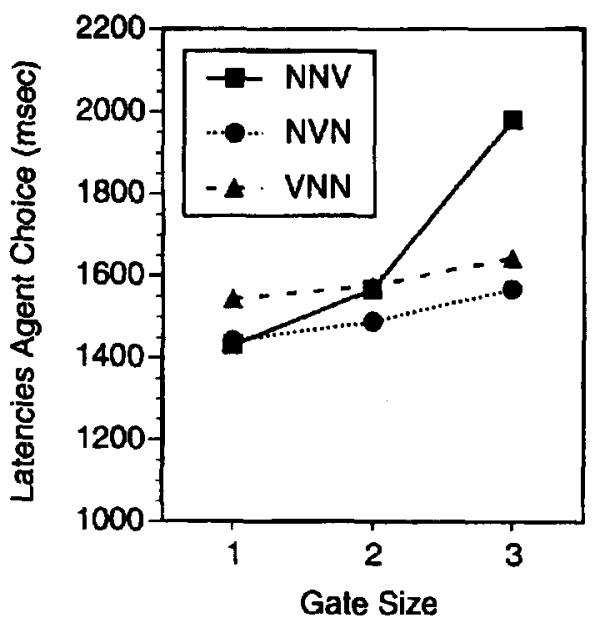

(b)

Figure 1. Choice responses (a) and reaction times (b) as a function of gate size and word order for the simple sentences (see note 1). 
reflected a competition between OSV and SOV, both of which are plausible interpretations for NNV in Chinese.

Note that for the NVN and NNV sentences, there was a bias toward the first-noun choice at the first gate. Instead of choosing both nouns randomly, listeners opted more often for the first noun. This bias is quite understandable, because upon hearing only the first noun, listeners will find it easier to select the first noun as the agent than to select another noun that has not appeared yet. In other words, the bias was toward what has been heard, in the absence of the alternative form in the input.

Most important, the significant interaction between word order and gate size $[F(4,76)=26.21, p<.001]$ reflected that listeners built up their interpretations in different ways for each sentence type. As can be seen in Figure 1a, firstnoun choice increased across gates for the canonical NVN sentences (from $76 \%$ on Gate 1 to $93 \%$ on Gate 3 ), while it decreased across gates for the other two order types (from $48 \%$ to $14 \%$ for VNN and from $77 \%$ to $54 \%$ for NNV). NNV and NVN sentences elicited the same choices at the first gate, indicating that the size of the first-noun bias was equivalent for these order types. But from this point on, the NVN sentences moved close to $100 \%$ certainty of noun choice whereas the NNV sentences moved toward chance. For the VNN sentences, the first gate consisted only of a single $\mathrm{V}$, and, naturally, responses were random on these items at the first gate. But a VO interpretation was reached as soon as the next piece of information came in, at which time listeners settled on a VO interpretation for the VN fragment (which was further confirmed when the final $\mathrm{N}$ was heard). These results indicated that for both NVN and $\mathrm{VNN}$, listeners became more certain about their choices with increasing information (on the basis of the canonical SVO and the VO interpretations), whereas for NNV, the opposite was true. The results are consistent with previous whole-sentence studies (especially P. Li et al., 1993), and moreover, they add additional information about the temporal structure within which decisions are made.

RT data. ANOVA on the RT data, as presented in Figure $1 \mathrm{~b}$, again showed significant main effects of word order $[F(2,38)=10.19, p<.001]$ and gate size $[F(2,38)=6.49$, $p<.01]$ and a significant interaction $[F(4,76)=19.52, p<$ $.001]$. Figure $1 \mathrm{~b}$ indicates that the locus of the interaction was in the difference between NNV and the other orders from Gate 2 to Gate 3. Post hoc tests indicated a significant difference between NNV and the other orders at Gate 3 (Tukey's HSD, $p<.05),{ }^{3}$ but no difference between NVN and VNN at all gates (Tukey's HSD, n.s.). Comparison with the choice data reveals that the increase in RT for NNV at Gate 3 was clearly due to the decision indeterminacy associated with the two possible interpretations of NNV: Whereas decision for NVN and VNN moved toward certainty as gate size increased, decision for NNV moved toward chance as more information came in.

Note that the effect of gate size is uninteresting by itself, because an incremental RT may reflect confounded effects both from properties of the sentence and from the length of the gate (longer materials simply take more time to process). However, the effect becomes interesting when we compare different word orders, within and across sentence gates. It also becomes interesting when we compare the relative RT increase with the relative size of the gate. For example, large increases such as NNV from Gate 2 to Gate 3 indicated that listeners were unable to make their decision even when all the information was received, but small increases relative to the size of the gate may indicate that listeners made a decision even before they had the complete sentence (e.g., in the NVN and VNN cases). In an analysis of listeners' response speed relative to gate length, it was found that the full NNV sentence elicited a response on the average $269 \mathrm{msec}$ after the end of the sentence (average length $=1,710 \mathrm{msec}$, average RT $=1,979 \mathrm{msec}$ ), but the full NVN and VNN sentences elicited responses before the end ( -180 and $-85 \mathrm{msec}$, respectively). When the amount of time involved in button pressing is considered, the results suggest that listeners initiated their responses for NVN and VNN before they were able to process the final word completely.

To summarize, the data from the simple sentences are consistent with data from earlier whole-sentence studies of Chinese (Miao, 1981; Miao et al., 1986; P. Li et al., 1993), reflecting a strong SVO interpretation for NVN, a strong VOS interpretation for VNN, and a chance-level interpretation between OSV and SOV for NNV. The results show how much can be learned from the sentence-gating method: Listeners start with default expectations, which are either confirmed or disconfirmed at subsequent gate points. Choices move toward certainty (for NVN and VNN) or toward the chance level (on NNV) depending on the upcoming information - that is, on the way in which word order cues unfold across the sentence. RT data complement and extend these analyses, allowing us to see how speed of processing is related to the clarity and certainty with which a decision can be made at different gate points: Both NVN and VNN lead to decisions before the end of the complete sentence, while the ambiguous NNV leads to decisions after the end of the sentence.

\section{Sentences With ba}

The design for this set of sentences was $3 \times 4 \times 3$ (word order $\times$ gate size $\times$ position of $b a$ ). Of all these combinations, the most canonical in the spoken language is the full sentence NbaNV. Other combinations are possible under certain pragmatic conditions (see Appendix B). Because of the number of cells involved in the design ( 36 in total), I will break down the analyses by word order types, looking first at choices and then RTs within each word order. A $3 \times 4 \times 3$ ANOVA on the data revealed that all the main effects and interactions (except the order $\times$ gate size effect in RT) were significant at $p<.05$.

NNV with $b a$. Figure 2 a presents the choice data, broken down by gate size and the position of $b a$. As can be seen, there was also a first-noun choice bias at Gate 1 for different position types, as in the simple sentences. This bias was most pronounced in the NbaNV sentences. ${ }^{4}$ Since $\mathrm{N} b a \mathrm{NV}$ was the canonical sentence for $b a$, later gates of this sentence also elicited the highest first-noun choices. In contrast, $b a \mathrm{NNV}$ elicited more second-noun choices 


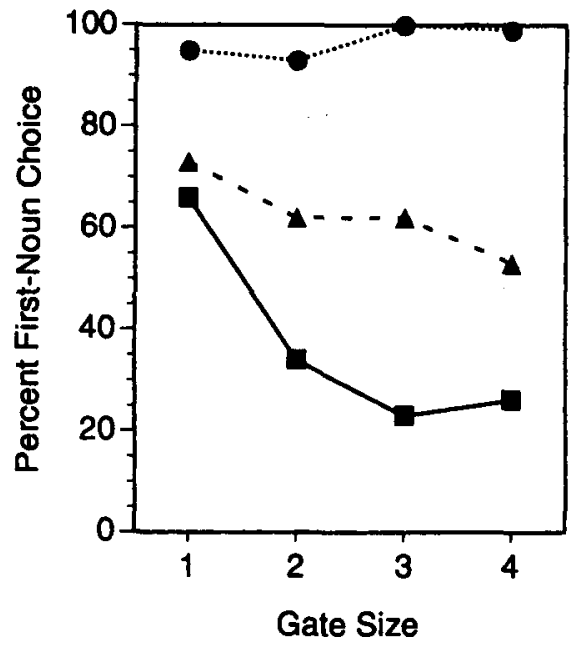

(a)

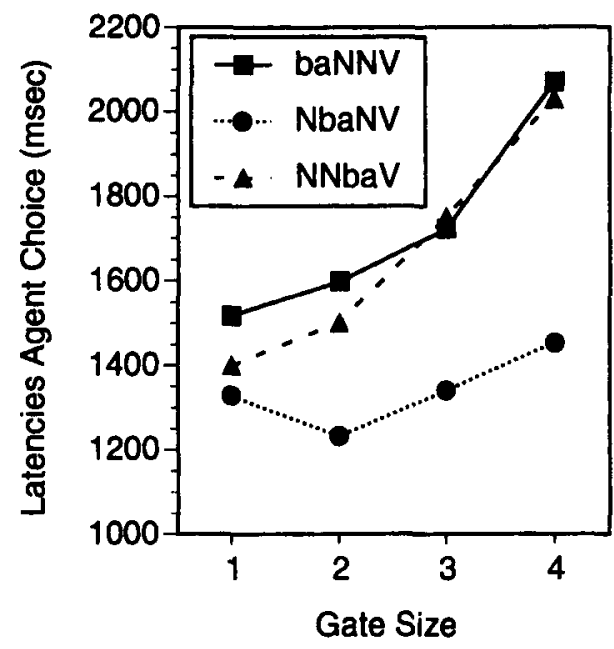

(b)

Figure 2. Choice responses (a) and reaction times (b) as a function of gate size and marker position for the ba sentences in the NNV order (see note 1 ).

after the first gate, because $b a$ marked the first $\mathrm{N}$ as the sentence patient and thus the second $\mathrm{N}$ could be interpreted as the agent. In between these two extremes was NNbaV, which was ungrammatical sincé $b a$ cannot occur before a verb in the language. Although the occurrence of $b a$ between $\mathrm{NN}$ and $\mathrm{V}$ produced a grammatical violation, it did not alter the basic pattern of interpretation with NNV sentences: The choice results (and RTs, too; see below) were very similar across different gates for $\mathrm{NN} b a \mathrm{~V}$ and the simple NNV without the $b a$ marker (see Figure la). This similar pattern indicated that the grammatical violation produced by the wrong positioning of the $b a$ marker did not cause interference to listeners' choice decisions nor their decision times in interpreting NNV.

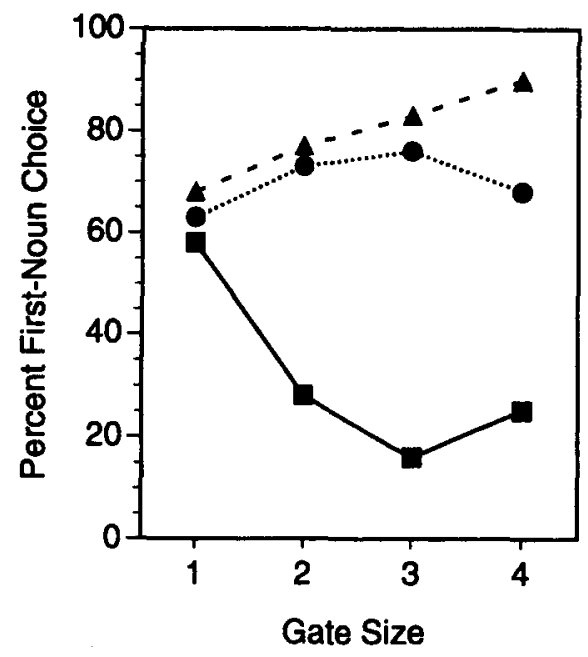

(a)
RT data for this set of sentences indicate, as shown in Figure $2 \mathrm{~b}$, that the canonical order $\mathrm{N} b a \mathrm{NV}$ elicited significantly faster responses than the other two types, but that the other two types were not different from each other (Tukey's HSD, n.s.). The general pattern for the RTs was an accelerating increase from gate to gate. This accelerating pattern was similar to that for the simple NNV sentences (see Figure 1b).

NVN with $b a$. Figure $3 a$ presents the choice data for this set of sentences. First, with $b a \mathrm{NVN}$, listeners began with a near-random choice ( $58 \%$ ) when hearing only the marker, but soon switched to a second-noun choice ( $78 \%$ and $84 \%$ at Gates 2 and 3, respectively). Up to this point, $b a \mathrm{NV}$ was a legal fragment from the canonical $\mathrm{N} b a \mathrm{NV}$; the

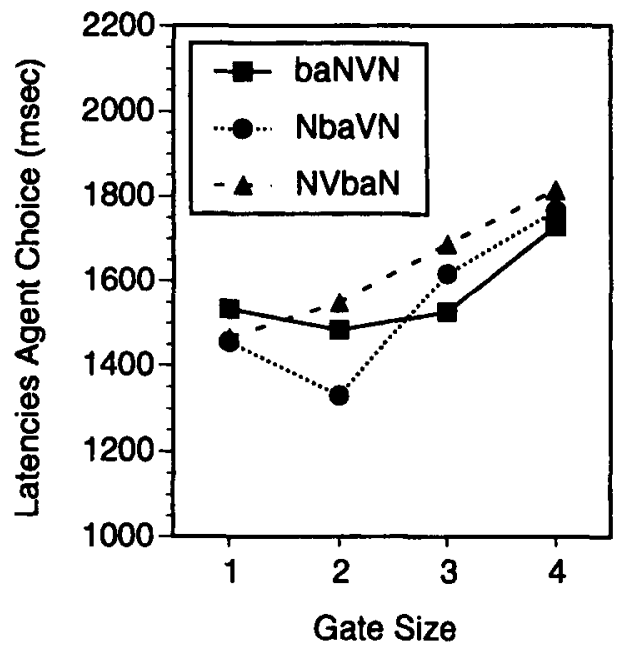

(b)

Figure 3. Choice responses (a) and reaction times (b) as a function of gate size and marker position for the $b a$ sentences in the NVN order (see note 1). 
$\mathrm{N}$ after $b a$ was marked as the object so that the alternative $\mathrm{N}$ in $b a \mathrm{NVN}$ could be assigned the agent role. However, when the final $\mathrm{N}$ actually appeared in $b a \mathrm{NVN}$, a competition emerged between $b a \mathrm{~N} / b a \mathrm{NV}$ and $\mathrm{VN}$, with both nouns competing for objecthood at the same time. Although the interpretation already established with the fragment $b a \mathrm{NV}$ won over VN to favor the second noun as the agent, the competition led to a slight increase in first-noun choice (from $16 \%$ to $25 \%$ at the end).

The above analysis brings us to an important point about the integration of fragment information in Chinese sentence comprehension: Listeners could interpret incomplete and ungrammatical sentences on the basis of partial overlap with complete and grammatical sentences in the language. The results with $b a \mathrm{NVN}$ showed that the fragment $b a \mathrm{NV}$ was interpreted as part of the complete canonical $\mathrm{N} b a \mathrm{NV}$, and when the final $\mathrm{N}$ appeared in $b a \mathrm{NVN}$, the fragment $\mathrm{VN}$ started to compete with $b a \mathrm{~N}$ or $b a \mathrm{NV}$. In Chinese, due to the properties of subject/object ellipsis and variable word order, it is not always clear which sentence is complete and which is incomplete. For instance, $b a \mathrm{NV}$ and VN can stand alone and be regarded as complete sentences with omitted subject, and NV as a complete sentence with omitted object or subject. Thus, Chinese listeners cannot afford to wait for "complete sentences" to occur in real-time processing, but have to integrate partial fragment information as soon as fragment interpretations become available.

Next, there was a general increase across gates in firstnoun choice for the NVbaN and NbaVN sentences, except for a final decrease for the latter (Tukey's HSD, n.s.). Note that for both NVbaN and NbaVN, the occurrence of $b a$ before or after the verb was ungrammatical because $b a$ needed to mark a preverbal noun. However, the increase in choices (especially with NVbaN) was similar to that with the simple NVN sentences in which there was no $b a$ marker, a result that again showed that the grammatical violations of $b a$ did not affect listeners' interpretations.

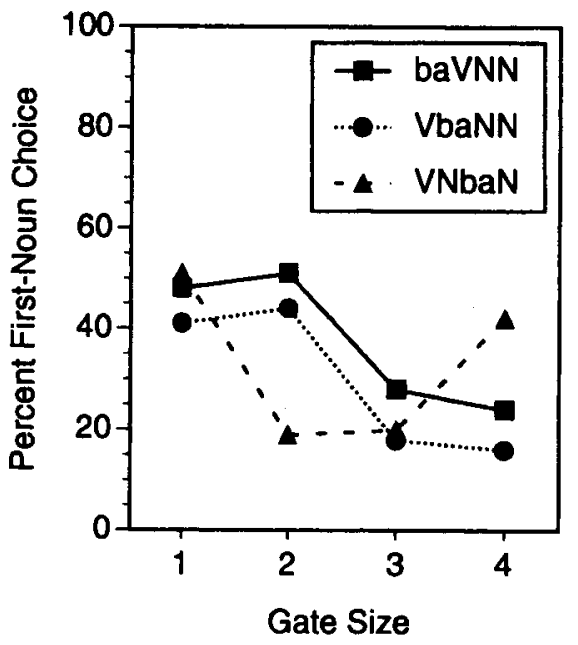

(a)
The RT data for the NVN sentences with $b a$, as presented in Figure 3b, reflected relatively small differences between the position types. Post hoc comparisons indicated no significant differences between any pair of the three position types. There was a decrease in RT from Gate 1 to Gate 2 for $\mathrm{N} b a \mathrm{VN}$, although this decrease was not statistically significant (Tukey's HSD, n.s.).

VNN with ba. As presented in Figure 4a, all choice data for this set of sentences fell into the lower half of the figure, showing that regardless of the position of $b a$, VNN sentences elicited predominantly second-noun choices. For all VNN sentences, at Gate 1 (plus Gate 2 for $b a \mathrm{VNN}$

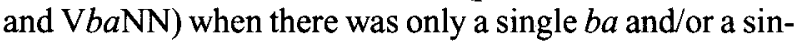
gle $\mathrm{V}$, listeners responded randomly or nearly randomly. But after this point (i.e., at Gate 1 for $\mathrm{VN} b a \mathrm{~N}$ and at Gate 2 for $b a \mathrm{VNN}$ and $\mathrm{V} b a \mathrm{NN}$ ), listeners' decisions moved quickly to the second $\mathrm{N}$ for agenthood. A noun following the verb, with or without $b a$, simply confirmed listeners' expectation about the object role of that noun. Thus, the increase in second-noun choice with these sentences was the same as that observed with the simple VNN sentences in which there was no $b a$ marker. In fact, with the first two gates collapsed, $b a \mathrm{VNN}$ and VbaNN had exactly the same pattern as the simple VNN. Again, $b a$ either preceding or following the verb did not affect listeners' interpretations, although in both cases the combination of $b a$ with the verb resulted in grammatical violation.

One other point concerning the choice data is that whereas the occurrence of the final $\mathrm{N}$ did not alter the previous choice in either $b a \mathrm{VNN}$ or $\mathrm{V} b a \mathrm{NN}$, it did so significantly in $\mathrm{VN} b a \mathrm{~N}$. In the former two cases, the final $\mathrm{N}$ was consistent with the already established interpretation about the object role of the first $\mathrm{N}$ and thus could be readily assigned the agent role. But in the latter case the final $\mathrm{N}$ created a strong competition between $\mathrm{VN}$ and $b a \mathrm{~N} / \mathrm{N} b a \mathrm{~N}$, leading to a significant increase in first-noun choice from Gate 3 to Gate 4 (Tukey's HSD). This com-

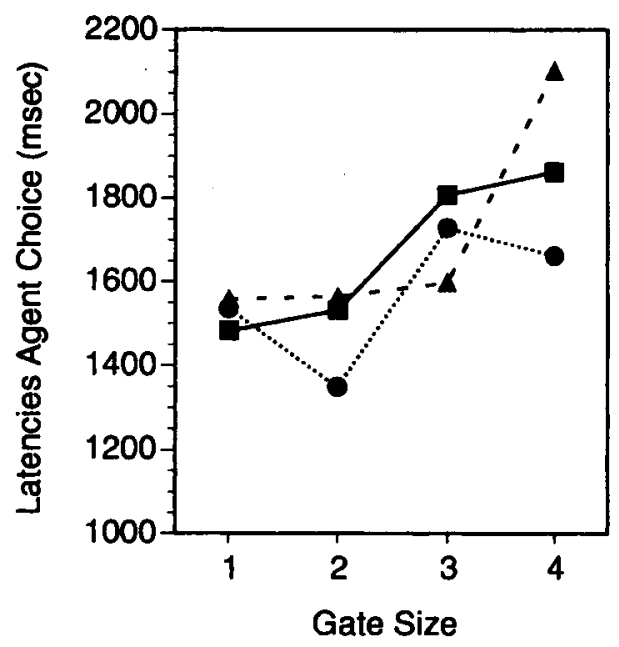

(b)

Figure 4. Choice responses (a) and reaction times (b) as a function of gate size and marker position for the $b a$ sentences in the VNN order (see note 1 ). 
petition also resulted in a sharp increase in RTs, as seen in Figure $4 b$.

Figure $4 \mathrm{~b}$ presents the RT data for the VNN sentences with $b a$. In addition to the significant increase from Gate 3 to Gate 4 with VNbaN (Tukey's HSD), two types of decreases in RT with the $\mathrm{VbaNN}$ sentences should be noted here: (1) the decrease from Gate 1 to Gate 2, and (2) the decrease from Gate 3 to Gate 4. These RT decreases showed significant differences between $b a \mathrm{VNN}$ and $\mathrm{V} b a \mathrm{NN}$ (post hoc tests indicated significant differences at Gates 2 and 4), although the two were the same in the choice data. For the first decrease, it is interesting to note that with all the $b a$ sentences, whenever $b a$ occurred at Gate 2 following a noun or a verb, the response was faster than that to the preceding noun or verb (see also Figures $2 b$ and $3 b$ ). This faster response, examined with the choice data, reflected that listeners simply held on to their previous interpretation, based either on first-noun bias or on random choice. They did not immediately integrate $b a$ with the previous information to make inferences about sentence roles probably because (1) no fragment interpretation was available without a noun following $b a$ (unlike bei, which could function without a following noun; see below); or (2) $b a$ was interpreted as a sentence-final particle rather than as an object marker (these two markers are homophonous in syllable though not in tone). As for the second decrease, the $b a$ marker and the order $\mathrm{VN}$ jointly pointed to the first $\mathrm{N}$ as the sentence patient, so that the second $\mathrm{N}$ could be more readily interpreted as the agent when it actually occurred in $\mathrm{V} b a \mathrm{NN}$. By contrast, in $b a \mathrm{VNN}$ there was no such combined effect on either the first or the second $\mathrm{N}$, and thus no such facilitation was found at the end.

To summarize, data from the $b a$ sentences suggest that Chinese listeners make important use of partial fragment information in spoken sentence comprehension. They can interpret incomplete fragments and ungrammatical sentences by reference to complete and grammatical sentences in the language. The stable patterns across the various simple and $b a$ sentences also show that grammatical violations in the $b a$ sentences do not affect Chinese listeners' interpretations of sentence roles. Because daily spoken Chinese contains many sentences that would be either incomplete or ungrammatical in English and other Western languages, and because grammatical functions are not overtly marked in Chinese as they are in those other languages, it is thus natural that Chinese listeners would rely on partial fragment information for sentence interpretation, and would show less sensitivity to grammatical violations. This is in sharp contrast to sentence comprehension in English, in which grammaticality plays a more important role (Bates, 1991; von Berger et al., in press).

\section{Sentences With bei}

The design for this set of sentences was also $3 \times 4 \times 3$ (word order $\times$ gate size $\times$ position of bei), the same as that for the $b a$ sentences. Of the various combinations, the natural orders include the full sentence NbeiNV (interpreted

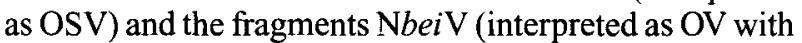
omitted S) and beiNV (interpreted as SV with omitted O). Other combinations are also possible under certain pragmatic conditions (see Appendix B). In line with the above analyses, I will break down the analyses by word order types, examining first choices and then RTs within each word order. A $3 \times 4 \times 3$ ANOVA on the data revealed that all the main effects and interactions were significant at $p<.05$.

NNV with bei. Figure 5a presents the choice data for this set of sentences. As can be seen, there was a first-noun bias at Gate 1 when only the noun had occurred, as with the $b a$ sentences. The choice pattern for $\mathrm{NN} b e i \mathrm{~V}$ was very similar to that for $\mathrm{NN} b a \mathrm{~V}$ (see Figure 2a): In both cases, the interpretation started with a first-noun bias and then lowered to nearly random choices $(57 \%$ for $\mathrm{NN}$ bei $\mathrm{V}$ and $53 \%$ for $\mathrm{NN} b a \mathrm{~V}$ at the final gate). In fact, the pattern was also similar to that for the simple NNV sentences without

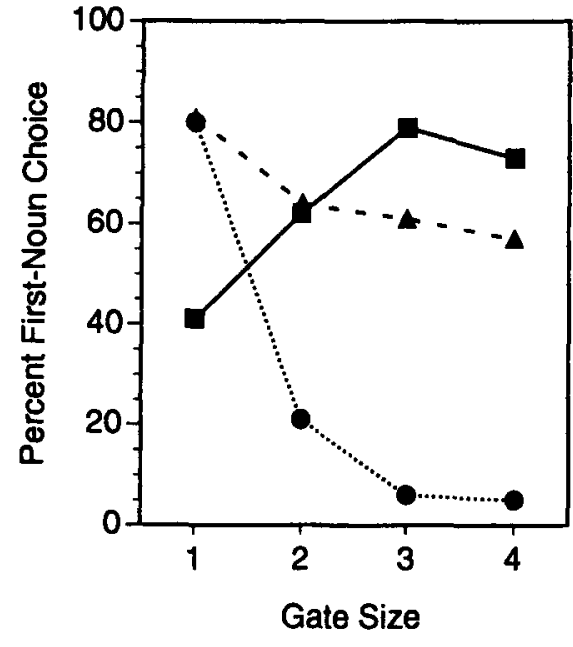

(a)

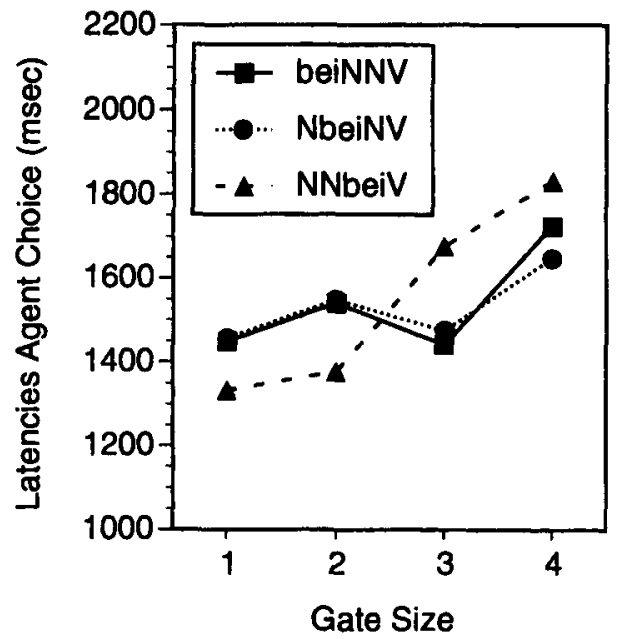

(b)

Figure 5. Choice responses (a) and reaction times (b) as a function of gate size and marker position for the bei sentences in the NNV order (see note 1). 
either of the markers (see Figure 1a). Again, the ungrammatical occurrence of bei before the verb, like $b a$ before the verb, did not disturb the listener's interpretation, although neither did it help the listener to resolve the ambiguity associated with the two possible interpretations of $\mathrm{NNV}$. In contrast to $\mathrm{NN} b e i \mathrm{~V}$, when bei occurred before a noun, as in bei NNV and Nbei NV sentences, the choice patterns differed greatly from the corresponding $b a$ sentences. First-noun choices for NbeiNV went from a high $80 \%$ to a very low $5 \%$, and for bei NNV, from a nearly random $41 \%$ to $73 \%$. These patterns were the reverse of those for the corresponding $b a$ sentences, naturally so, because $b a$ and bei pointed to different directions for agenthood: The former marked the subsequent noun as the sentence patient, while the latter marked the same noun as the sentence agent.

Earlier analyses of the $b a$ sentences indicated that the canonical NbaNV elicited the most uniform choice decisions ( $99 \%$ first-noun choice at the final gate). Similarly, for the bei sentences, the canonical Nbei NV also elicited highly consistent choices $(95 \%$ second-noun choice at the final gate). Bei, like $b a$, when inserted before the second $\mathrm{N}$ preverbally, serves to disambiguate the two possible interpretations of NNV. However, when inserted before the first $\mathrm{N}$, as in bei $\mathrm{NNV}$, the sentence elicited only moderately consistent choices ( $73 \%$ first-noun choice at Gate 4 ). In bei $\mathrm{NNV}$ there was also a competition between bei $\mathrm{N}$ and $\mathrm{NV}$, in which both nouns were competing for agenthood, leading to a decrease in first-noun choice from Gate 3 to Gate 4 (and increases in RTs; see Figure 5b).

The RT data for this set of sentences, as presented in Figure $5 \mathrm{~b}$, further complemented the analyses of the choice data. The overall linearly increasing pattern for NNbei $\mathrm{V}$ was similar to the patterns for both simple $\mathrm{NNV}$ and $\mathrm{NN} b a \mathrm{~V}$ sentences and was consistent with the choice data in which NNV sentences had the same choice patterns, with or without the markers. The significant increase from Gate 2 to
Gate 3 (Tukey's HSD) reflected the listener's puzzle about the same ambiguity associated with NNV: Upon hearing two Ns consecutively, the listener did not know which would be the sentence agent, and neither a following bei or V could help. Figure $5 \mathrm{~b}$ also shows that the pattern for NNbei $\mathrm{V}$ was different from the patterns for bei NNV or NbeiNV. The patterns for beiNNV and NbeiNV were essentially the same, a result that could be due to the similar decision certainty associated with the two types of sentence as decisions moved across the sentence: There were gradually more first-noun choices for be $i \mathrm{NNV}$, but more second-noun choices for NbeiNV (the same picture can be seen in Figures $6 \mathrm{a}$ and $6 \mathrm{~b}$ for the NVN sentences).

NVN with bei. Figure 6a presents the choice data for this set of sentences. First, the choices for be $i \mathrm{NVN}$ started with a random choice at Gate 1 when only bei was given, but then quickly moved to a first-noun choice. This pattern was the opposite of that for $b a \mathrm{NVN}$, because the fragments bei $\mathrm{N}$ and $\mathrm{VN}$ converged on the first noun as the sentence agent, whereas the two nouns in $b a \mathrm{~N}$ and $\mathrm{VN}$ competed for objecthood. Second, both NVbeiN and NbeiVN (especially the former) had a first-noun bias at Gate 1 when only the $\mathrm{N}$ had occurred. For NVbei $\mathrm{N}$, the bias was further confirmed when the listeners heard NV, leading to a very high $98 \%$ first-noun choice. But when the listeners heard bei after NV, the first-noun choice lost weight, since bei would indicate a following noun as the sentence agent. The weight went down further to favor a second-noun choice when the final $\mathrm{N}$ appeared, although not completely due to the competition between NV and beiN, in which both nouns were competing for agenthood. Finally, for NbeiVN, the decision moved quickly from a first-noun bias to a second-noun choice when bei occurred, and the secondnoun choice continued through to the final gate. Note that the occurrence of bei in Nbei $\mathrm{VN}$, unlike that of $b a$ in $\mathrm{N} b a \mathrm{VN}$, strongly affected the SVO interpretation associated with NVN. This difference between $b e i$ and $b a$ is because in the

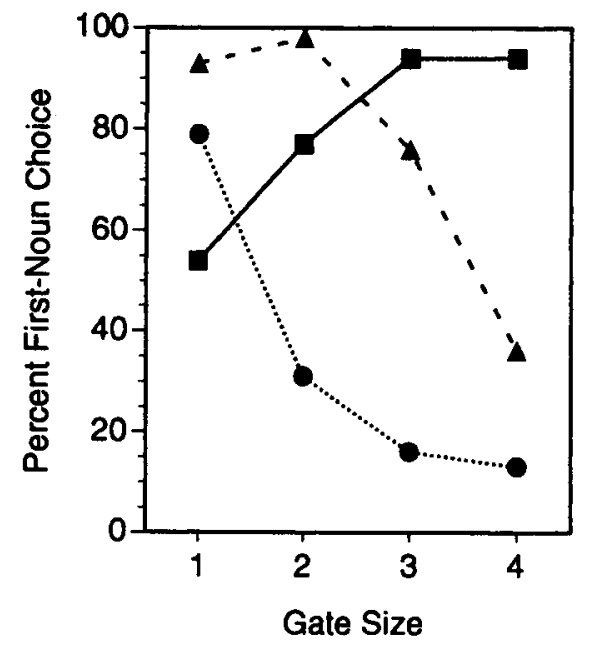

(a)

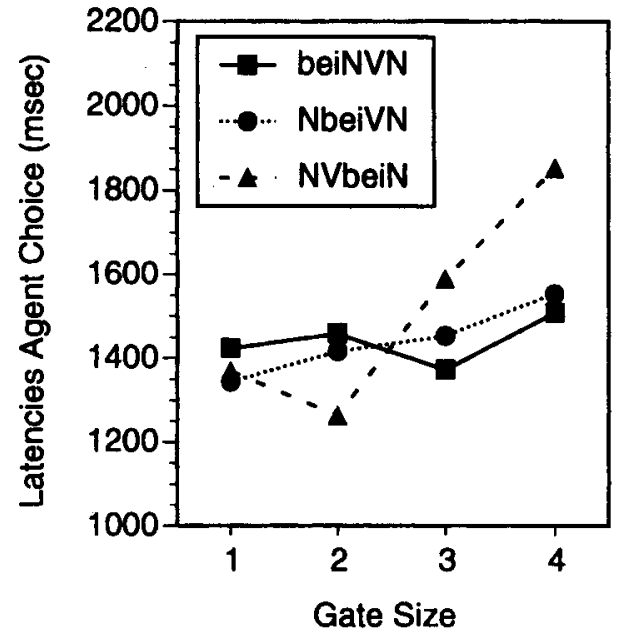

(b)

Figure 6. Choice responses (a) and reaction times (b) as a function of gate size and marker position for the bei sentences in the NVN order (see note 1 ). 
adult language the sentence agent can be legitimately omitted from Nbei NV (resulting in the fragment Nbei V, similar to truncated passives in English), whereas the noun after $b a$ in NbaNV cannot be legally omitted. Thus, listeners

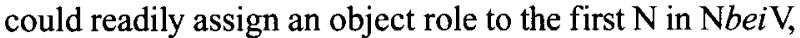
using fragment interpretation based on the complete form $\mathrm{Nbei} \mathrm{NV}$. When the final N in Nbei VN appeared, it would simply be mapped onto the already-established secondnoun choice interpretation.

Related to the $b a$ and bei difference is that the occurrence of $b a$ usually did not have an immediate effect on listeners' interpretation, as shown in the earlier discussion, but the occurrence of bei did. For example, the occurrence of bei in both $\mathrm{Nbei} \mathrm{VN}$ and $\mathrm{NV}$ bei $\mathrm{N}$ immediately changed the choice patterns of the sentence, leading to a sharp decrease in first-noun choice. This was also true of the Nbei NV sentences discussed earlier.

What led to the discrepancy between $b a$ and $b e i$ ? P. Li et al. (1993) have argued that the two markers have different weights in determining sentence roles, on the basis of the syntactic, semantic, and phonological properties of $b a$ and bei. Bei is uniformly associated with the function of passive marking (e.g., it can directly mark the verb in $\mathrm{N} b e i \mathrm{~V}$ ), but the validity of $b a$ as a pure object marker is reduced by its multifunctionality in the language (e.g., its marking of other sentence roles and its homophony with other particles; see earlier discussion). Thus, it is not surprising that listeners relied more strongly on the bei marker than on the $b a$ marker, as found in P. Li et al., and that $b e i$ but not $b a$ had an immediate effect on choice decisions, as shown in these experiments.

Figure $6 \mathrm{~b}$ presents the RT data for the NVN sentences with bei. As can be seen, a major difference existed between $\mathrm{NV} b e i \mathrm{~N}$ on the one hand and bei $\mathrm{NVN}$ and $\mathrm{Nbei \textrm {VN }}$ on the other, while beiNVN and Nbei VN themselves were not different from one another (Tukey's HSD, n.s.). The decrease in RT from Gate 1 to Gate 2 for NVbei $\mathrm{N}$ was con- sistent with the choice patterns in which the unfolding of the NV order confirmed the initial first-noun choice. The increase in RT from Gate 2 to Gate 4 was clearly due to the competition between $\mathrm{NV}$ and be $\mathrm{N}$, in which the word order and the $b a$ marker pointed to different directions for agenthood. On the other hand, the relatively flat patterns with bei $\mathrm{NVN}$ and $\mathrm{N}$ bei $\mathrm{VN}$ showed that these sentences elicited fast responses, especially when the RTs were considered against gate size. In an analysis of RT relative to gate length, bei NVN elicited responses on the average $296 \mathrm{msec}$ before the end of the final gate (gate length $=1,803 \mathrm{msec}$, average $\mathrm{RT}=1,507 \mathrm{msec}$ ), and $\mathrm{Nbei} \mathrm{VN}$ elicited responses on the average $217 \mathrm{msec}$ before (gate length $=1,770 \mathrm{msec}$, average $\mathrm{RT}=1,553 \mathrm{msec}$ ). These results were consistent with the choice data, indicating that listeners had settled on a firm interpretation before the final gate (Figure 6a).

VNN with bei. As presented in Figure 7a, in the choice data, both beiVNN and VbeiNN started with nearly random choices and stayed roughly at that level at the second gate, when only a $\mathrm{V}$ and/or bei had occurred. This pattern was similar to that for the corresponding $b a$ sentences. A single marker either before or after the verb did not help the listener to assign sentence roles, when no information about the noun phrase was actually presented. However, after the initial gates, bei VNN and VbeiNN started to have different patterns, unlike $b a \mathrm{VNN}$ and $\mathrm{V} b a \mathrm{NN}$, which were essentially the same across gates (Figure 4a). For bei $\mathrm{VNN}$, the choices went down a little below the chance level, slightly favoring the second $\mathrm{N}$ as the sentence agent ( $64 \%$ second-noun choice at the final gate). This result reflected a reduced VOS interpretation, because the fragment interpretation associated with bei $\mathrm{V}$ conflicted with VNN for VOS (bei V suggested an omitted object before bei and thus would compete with a following VO). For VbeiNN, the choices went up to favor the first N (69\% first-noun choice at the final gate), because bei marked the first $\mathrm{N}$ as sentence agent. But it did not result in consistent choices

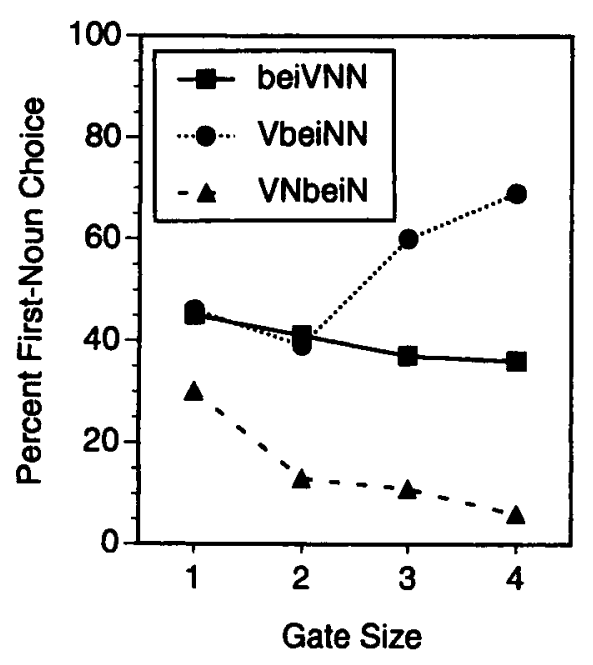

(a)

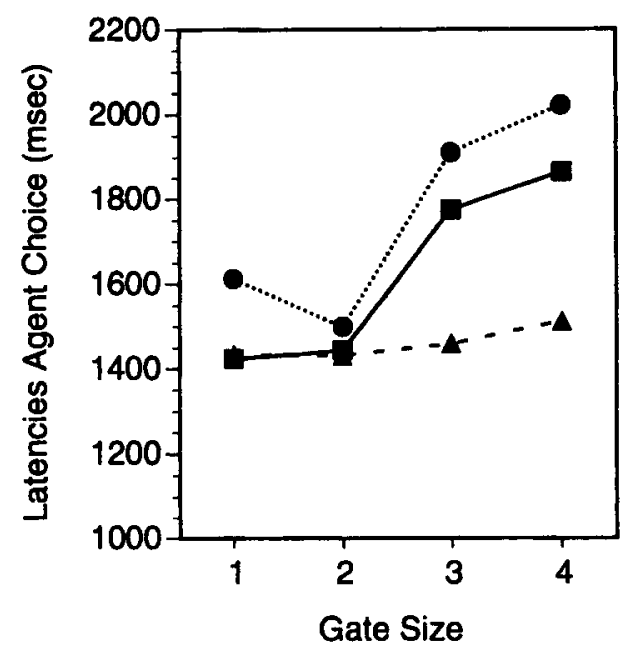

(b)

Figure 7. Choice responses (a) and reaction times (b) as a function of gate size and marker position for the bei sentences in the VNN order (see note 1). 
either, due to the competition between the fragment interpretations associated with be $i \mathrm{~N}$ and the $\mathrm{VN}$ order.

In contrast to bei $\mathrm{VNN}$ and $\mathrm{V} b e i \mathrm{NN}$, consistent and uniform second-noun choices were derived for $\mathrm{VN} b e i \mathrm{~N}$, in which VN and bei $\mathrm{N}$ converged to point to the first $\mathrm{N}$ as the patient and the second $\mathrm{N}$ as the agent. VNbei $\mathrm{N}$ started with a second-noun choice bias when only the $V$ had occurred (which was found to be due mainly to the "animateness" effect of the noun phrases; see note 4). And this bias was confirmed by subsequent information, leading to a uniform $94 \%$ second-noun choice at the final gate.

Figure $7 \mathrm{~b}$ shows that the RT data were largely consistent with the choice data. The convergence VNbei $\mathrm{N}$ case differed significantly from the competition cases bei VNN and VbeiNN, but the two competition cases did not differ from each other (Tukey's HSD, n.s.). The convergence VNbeiN elicited significantly faster responses than the competition cases bei $\mathrm{VNN}$ and $\mathrm{Vbe} i \mathrm{NN}$ from Gate 2 onward: The former showed no clear increase across gates, whereas the latter two increased sharply from Gate 2 to Gate 4 . In fact, VNbei $\mathrm{N}$ elicited responses even before the final gate (at an average of $-223 \mathrm{msec}$ ) in an analysis of RT relative to gate length.

To summarize, the choice data and the RT data from the bei sentences provide information consistent with and complementary to the moment-to-moment processes involved in listeners' interpretation of the bei sentences. The results are also consistent with those from the simple and the $b a$ sentences. In particular, listeners use fragment interpretations for both grammatical and grammatically violated forms, and inside these forms word order cues and the bei marker interact (either compete or collaborate) to determine patterns of choice decision and decision time as the cues unfold across the sentence. Consistent or converging cues lead to uniform choices and faster RTs, while competing cues lead to diverse choices and slower RTs. The above analyses have also shown some important differences between $b a$ and $b e i$ in the weights and the immediacy of effects with which they can influence spoken sentence comprehension in Chinese. These differences are related to the clarity of the marker's function in designating sentence roles.

In sum, using the sentence-gating method in this experiment, I have obtained consistent and converging results from all three sets of sentences regarding the relationship between sentence role assignment, processing speed, and cue interactions. Gating provides a useful way of looking into the comprehension process as the auditory input accumulates over time. It allows us to see the temporal structure involved in this process, for example, by showing how choice decisions move along the course of the sentence (decision is not a one-time event) and how decision times change across gate points as a function of the convergence and competition between different cues (e.g., word order and $b a$ and $b e i$ in Chinese). Because gating also permits us to evaluate listeners' comprehension of a sentence on the basis of partial or fragment information for that sentence, results from this experiment add to our understanding about the role of partial information and fragment interpretation in spoken sentence comprehension. For example, a given sen- tence such as $b a \mathrm{NVN}$ is associated with multiple fragment interpretations including $b a \mathrm{~N}, \mathrm{NV}, \mathrm{NVN}$, and VN in Chinese, and gating provides a means of probing into these interpretations. The results indicate that Chinese listeners rapidly integrate partial sentence information as soon as fragment interpretations become available, and they interpret incomplete and ungrammatical sentences by using native comprehension strategies relevant for the analysis of the particular structure. The results also indicate that grammatical violations in $b a$ and $b e i$ sentences do not affect listeners' interpretation of sentence roles. These features in processing largely reflect influences of language-specific properties of Chinese in which morphological, grammatical, and syntactic constraints on sentence constituents are very weak.

\section{EXPERIMENT 2}

Results from Experiment 1 attest to the usefulness of the sentence-gating method. Although gating has been used successfully in many studies of spoken word recognition, questions have been raised about the possible strategic, nonlinguistic effect of the way in which gates are successively presented to listeners (see Grosjean et al., 1994). Grosjean and his colleagues have put some of these doubts to rest by showing that successive and individual presentation formats lead to the same results, and that data from gating correlate highly with data obtained with other on-line tasks such as word monitoring, naming, and cross-modal priming (Cotton \& Grosjean, 1984; Marslen-Wilson, 1990; Tyler \& Wessels, 1985). To forestall similar questions in the context of spoken sentence comprehension, I designed two more experiments, Experiment 2, which presented sentence gates individually and randomly, and Experiment 3 , which used a naming task, to examine the same variables as those of Experiment 1. A correlation of the data from these experiments with those from Experiment 1 would provide further evidence for using sentence gating as a tool in tapping into sentence comprehension processes.

\section{Method}

\section{Participants}

Twenty native adult Mandarin Chinese speakers participated in this experiment $(15$ males and 5 females, mean age $=30)$. They were matched with the participants in Experiment 1 for their language background. None had taken part in Experiment 1.

\section{Materials and Experimental Apparatus}

The same materials and apparatus as those used in Experiment 1 were used in this experiment.

\section{Procedure}

All procedures were the same as in Experiment 1, except the following. In Experiment 1, sentence gates were presented successively in a sequential order, from the shortest to the longest. In this experiment, these same gates were presented in a completely random order, and the individual gates from different sentence types were also intermixed. It was not possible to predict at any given point which sentence gate would occur next.

\section{Data Analysis}

The same two dependent variables, choices and RTs, were measured in this experiment in the same way as in Experiment 1 (see 
Appendix $\mathrm{C}$ for means of all data cells). ANOVAs on the three sentence types (simple, $b a$, and $b e i$ ) revealed that all the main effects and interactions were significant at $p<.05$. Although detailed analyses of the individual patterns are available, as in Experiment $1,{ }^{5}$ for the sake of brevity, I will report only results from correlational analyses on the two experiments to compare how data from successive and individual presentations may be correlated.

\section{Results and Discussion}

Several correlation analyses were conducted on the two sets of data from the two experiments, treating the individual cells ( 9 for simple, 36 for $b a$, and 36 for $b e i$ ) as subjects in the analysis. The Pearson product-moment correlation coefficients are summarized in Table 1 , for choices and RTs, respectively.

These results indicate very high correlations between the data from the two experiments, for both choices and RTs, although the RT data had relatively lower correlations than did the choice data. In this experiment the successive and repetitive aspects of the gating task were completely eliminated by the random presentation of individual gates. Listeners at any given point of the sentence could not utilize information from the previous gate in the same fashion as they could (if at all) in a standard gating task. The high correlations between data from the two experiments suggested that the patterns observed in Experiment 1 were not particularly due to the successive presentation format of the gating method. These results are also consistent with observations of spoken word recognition by Cotton and Grosjean (1984), who compared successive and individual presentations of word gates and found consistent results with both presentation formats.

To summarize, Experiment 2 provided further support for the sentence-gating method for studying the temporal structure of spoken sentence comprehension and has shown converging evidence for the patterns observed in Experiment 1 regarding the relationship between cue interaction and the time course of sentence comprehension.

\section{EXPERIMENT 3}

In spoken word recognition there is evidence that gating results correlate highly with results from naming studies (Marslen-Wilson, 1990; Tyler \& Wessels, 1985). To further

Table 1

Correlations Between Experiments 1 and 2 for Different Sentence Types

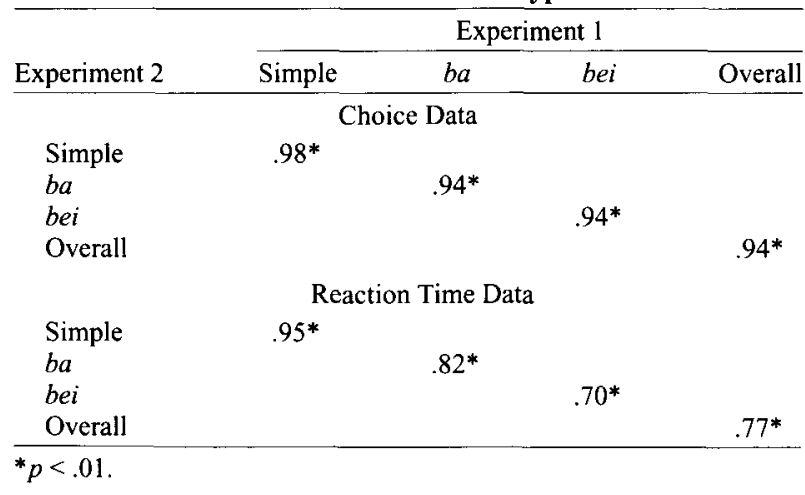

verify the findings from Experiments 1 and 2, I employed an agent-naming task in Experiment 3. Because naming is a simple and natural task, it is generally considered to be a truly on-line task. Thus, consistent data from this task could provide further support for the sentence-gating method.

The agent-naming task utilized here is a variant of the word-naming or shadowing/repetition task used by MarslenWilson (1985), and more recently by Liu et al. (in press) and Slowiaczek (1994). In the single-word shadowing task used by Liu et al., listeners were required to repeat the target word embedded in a sentence as soon as possible, and the target word was pronounced by a voice of the opposite sex from that in which the sentence had been pronounced. In the agent-naming task used here, it was not necessary to change the voice pronouncing the target word, since the target word (i.e., the agent) was to be identified by the listener. The listener's job was simply to name the sentence agent aloud as soon as possible upon hearing the sentence.

\section{Method}

\section{Participants}

Twenty native adult Mandarin Chinese speakers participated in this experiment ( 7 females and 13 males, mean age $=33$ ). They were matched with the participants in Experiments 1 and 2 for their language background. None had taken part in Experiment 1 or 2.

\section{Materials and Experimental Apparatus}

The same materials and apparatus used in Experiments 1 and 2 were used in this experiment. In Experiments 1 and 2, however, listeners were presented with sentence gates of various size, whereas in this experiment listeners were presented with whole sentences only for naming. This modification eliminated the sentence gate variable, and thus only word order and the position of the marker were involved in the experimental design (three orders for the simple sentences, and three orders $\times$ three positions for the $b a$ and $b e i$ sentences). There was a total of 108 sentences in the experiment ( 36 each for simple, $b a$, and $b e i$ sentences).

\section{Procedure}

During the experiment, listeners heard each sentence through a pair of headphones and simultaneously saw on the computer screen a pair of pictures that corresponded to the two nouns described in the sentence. Their task was to repeat aloud into a microphone the word that indicated the sentence agent, as quickly and as accurately as possible. A SONY high-impedance microphone was connected to the CMU button box through the voice-activated relay of the box. Auditory input from the microphone triggered the internal oscillator of the button box, and the response latencies were recorded by the PsyScope program. Listeners were given a maximum of $2 \mathrm{sec}$ to respond after the offset of the sentence. The overall miss rate was $4 \%$, indicating that the 2-sec timeframe was sufficient for most listeners to give their responses, while at the same time putting them under time pressure.

Before the experiment, listeners were familiarized with printed pictures of the animals that they would see during the experiment. The pictures were printed in the same size and shading as those in which they had appeared on the computer screen during the experiment. Listeners were also asked to repeat aloud the names of each of these animals during the familiarization process. They heard the 108 test sentences randomly in two blocks, with a 5-min break in between. The experiment lasted about $25 \mathrm{~min}$.

\section{Data Analysis}

The dependent variables were the same as those in Experiments 1 and 2. RTs were measured from the onset of the sentence to the onset 
Table 2

Pairwise Correlations for Data From the Three Experiments

\begin{tabular}{clc}
\hline Experiments & Data & Pearson $r$ \\
\hline 1 vs. 3 & Choice & $.93^{*}$ \\
2 vs. 3 & Choice & $.94^{*}$ \\
1 vs. 3 & RT & $.71^{*}$ \\
2 vs. 3 & RT & $.77^{*}$ \\
\hline
\end{tabular}

Note-RT, reaction time. ${ }^{*} p<.01$.

of the listener's vocal response (see Appendix D for means of all data cells). ANOVAs on the three sets of sentences (simple, $b a$, and $b e i$ ) revealed that all main effects and interactions were significant at $p<$ .05 , except for the word order main effect in the bei sentences. ${ }^{6}$

\section{Results and Discussion}

To highlight the major concern here, I will focus on comparing results from the current experiment with those from the sentence-gating experiments by using correlational analyses. To do this, I conducted pairwise correlations on the three sets of data from the three experiments. Because the naming experiment contained one fewer variable (the gate size variable), I pulled out only those cells from Experiments 1 and 2 (i.e., the cells from the last gate) that matched with cells from Experiment 3 for the analysis. Therefore, for each pairwise correlation, all data cells from one experiment were paired with all the corresponding cells from the other experiment (three simple, nine $b a$, and nine be $i$ in each experiment). Table 2 summarizes the results for both choices and RTs.

These analyses showed very high correlations between data from the three experiments, although the choice data again had higher correlations than did the RT data. Thus, the agent-naming results provided further support for the patterns observed in the sentence-gating experiments. The consistency between these experiments also converged with studies in spoken word recognition, in which a close relationship between gating and naming was observed (P. Li, in press; Marslen-Wilson, 1990).

To sum up, converging evidence was found in three experiments for both the usefulness of the sentence-gating method and for the analyses of the temporal structure of spoken sentence comprehension in Chinese. Results from the above experiments also complement previous wholesentence studies showing that listeners' decisions on sentence roles are constrained by the way in which different cues interact during the course of the sentence, and that decision times reflect degrees of competition and convergence between different cues. In general, consistent cues lead to uniform choice decisions and faster decision times, whereas competing cues lead to diverse decisions and slower decision times at various temporal locations of the sentence.

\section{GENERAL DISCUSSION}

A basic mechanism assumed here about spoken sentence comprehension is the parallel activation of multiple information sources and the competition between these sources, as assumed in various interactive activation models of word recognition and sentence processing (Marslen-
Wilson, 1987; McClelland, Rumelhart, \& the PDP Research Group, 1986; MacWhinney \& Bates, 1989). Spoken sentence comprehension can be viewed as a process in which the listener continuously evaluates information that is both currently available and yet to come in; the way these types of information interact determines the speed of interpretation. For example, as soon as a transitive verb is heard, the listener may simultaneously activate related pieces of information such as (1) there will be a noun phrase following the verb and (2) the noun phrase will be the patient of the action. Both expectations will be confirmed when the noun occurs in the VO structure, and the listener readily and quickly interprets the fragment or sentence. If some other constituents occur in place of the noun, the expectations will be disconfirmed, resulting in confused decisions and slower decision times. But the disconfirmation can also activate new expectations for subsequent input. These processes correspond closely to the processes in spoken word recognition, in which listeners activate different word candidates during the course of recognition (Grosjean, 1980, 1988; $\mathrm{P}$. Li, in press).

Parallel activation and interaction lead to fast interpretation of sentence meanings. Studies in spoken word recognition show that in normal context, listeners are able to identify a word when only half or even less of the acousticphonetic information of the word is perceived (Grosjean, 1980; Marslen-Wilson, 1987). Results from the current experiments also indicate that for many sentences in which the cues are consistent, listeners can make a decision about sentence roles before the complete sentence is heard. The results provide some empirical evidence for our intuition that spoken sentence comprehension often takes place well before the end of the complete signal. ${ }^{7}$

It should be noted that sentence comprehension differs from word recognition in that the former deals with an infinite number of sentence items, whereas the latter deals with a finite set of words. This difference leads to some differences in the task of the two processes. In sentence comprehension, the listener's task is to interpret the relationships between different sentence constituents, for example, to decide who does what to whom, whereas in word recognition, the listener's task is to access lexical entries in the mental lexicon and select a particular entry's unique phonological shape and semantic content. Nevertheless, the task differences should not prevent some processing similarities that may hold between the lexical processor and the sentence processor (in contrast to the view that the lexical processor is fundamentally different from the sentence processor; see, e.g., Frazier, 1990). The sentence processor, like the lexical processor, can similarly activate relevant sources of information in parallel and assess their status in the form-meaning mapping process, so that sentence roles may be evaluated on the basis of both available and predicted information (see MacDonald, Pearlmutter, \& Seidenberg, 1994, for a similar argument about the relationship between lexical and syntactic processing). Fragment interpretation in Chinese provides a good example for this sort of process: At a given temporal location in a sentence, there might be multiple fragment interpretations for that sentence. 
The rapid integration of sentence fragments in Chinese sentence comprehension largely reflects properties of the Chinese language. Chinese is well known for its lack of grammatical morphology. In contrast to most Indo-European languages, Chinese makes no use of grammatical devices such as number, gender, tense, or case to indicate relations between nouns or between nouns and verbs. In other words, grammatical functions and relations between major sentence constituents are not linked by morphological associations. Moreover, Chinese permits a large range of word order variations (see earlier discussion). The lack of inflections, the variability of word order, and the high degree of ellipsis mean that many daily sentence constructions in spoken Chinese would be incomplete and/or ungrammatical in English and other Western languages. These properties indicate that morphological, grammatical, and syntactic constraints in Chinese are very weak.

Given these properties, in order to efficiently and effectively pick up the relations between nouns or nouns and verbs, the Chinese listener has to rely on a processor that rapidly integrates interpretations associated with partial, fragmentary information (e.g., the various word order options, the $b a$ and $b e i$ structures). Although rapid interpretation characterizes sentence processing in many languages (e.g., as with the parsing of English garden-path sentences; see Frazier, 1987), this characteristic might be particularly relevant in Chinese. The Chinese listener cannot afford to wait until the "complete sentence" occurs and then start processing because Chinese is a language where a great deal of daily spoken sentences are incomplete or where it is not even clear what is complete and what is not. Because grammatical functions and relations are not overtly marked by morphological and syntactic devices in Chinese, as they are in most Indo-European languages, it should also come as no surprise that in realtime sentence comprehension, Chinese listeners rely more on fragment interpretations and on the semantic contents and relations of major sentence constituents (see also P. Li et al., 1993), but show less sensitivity to grammatical relations and grammatical violations.

Support for the last point can also be found in related research testing both grammatical and ungrammatical sentences in Chinese, English, and Chinese-English bilingual situations. In a separate analysis of the data reported in P. Li et al. (1993), I found that grammaticality was among the weakest factors affecting listeners' interpretation of sentence roles in Chinese. By contrast, in an analysis of monolingual English comprehension data, Bates (1991) showed that grammaticality is an important factor that affects English listeners' sentence interpretation in both choice decisions and RTs (some of the data are reported in von Berger et al., in press). Finally, in Liu et al. (1992a), experiments with bilingual English-Chinese speakers indicated that native Chinese listeners can separate grammaticality from interpretation: They may judge a particular sentence to be ungrammatical, but they can still process the sentence in a normal way, using native comprehension strategies that are relevant for the analysis of grammatical sentences. In the same study, English learners of Chinese experienced great uncertainty in their use of processing strategies when confronted with the same sentences.

Theories of linguistics have assigned a central role to grammaticality and syntactic structure. Meaningless sentences like "colorless green ideas sleep furiously" are used to demonstrate that it is possible for the listener to judge the grammaticality and understand the structure of a sentence without retrieving its meaning (Chomsky, 1957). This central role certainly has its place in many Indo-European languages, but whether it has the same place in Chinese and the same implications for Chinese processing is as yet unclear. Data from the present study suggest that listeners can retrieve the meaning of a sentence without being disturbed by grammatical violations, and that partial structures or fragments can lend themselves to probabilistic interpretations on the basis of complete and grammatical models in the language. There is not yet sufficient evidence to say that spoken sentence comprehension in Chinese is fundamentally different from that in other languages, but there is good reason to believe that at least the way in which the sentence processor operates on particular linguistic materials may differ from language to language due to influences from language-specific properties (e.g., in early vs. late decision making, in the utilization of fragment information, and in the degree of sensitivity to grammatical violations).

\section{REFERENCES}

Aaronson, D., \& Ferres, S. (1986). Sentence processing in ChineseAmerican bilinguals. Journal of Memory \& Language, 25, 136-162. BATES, E. (1991). On-line studies of sentence processing and the competition model. Unpublished manuscript, University of California, San Diego.

BATES, E., \& MACWhinNeY, B. (1982). Functionalist approaches to grammar. In E. Wanner \& L. Gleitman (Eds.), Language acquisition: The state of the art (pp. 173-218). New York: Cambridge University Press.

CHAO, Y.-R. (1968). A grammar of spoken Chinese. Berkeley: University of California Press.

Сномsкy, N. (1957). Syntactic structures. The Hague: Mouton.

Cohen, J., MacWhinney, B., Flatt, M., \& Provost, J. (1993). PsyScope: A new graphic interactive environment for designing psychology experiments. Behavior Research Methods, Instruments, \& Computers, 25, 257-271.

Cole, P. (1987). Null objects in universal grammar. Linguistic Inquiry, 18, 597-612.

ComRIE, B. (1989). Language universals and linguistic typology: Syntax and morphology (2nd ed.). Oxford: Blackwell.

Cotton, S., \& Grosjean, F. (1984). The gating paradigm: A comparison of successive and individual presentation formats. Perception \& Psychophysics, 35, 41-48.

FraziER, L. (1987). Sentence processing: A tutorial overview. In M. Coltheart (Ed.), Attention and performance XII (pp. 559-586). Hillsdale, NJ: Erlbaum.

FrAZIER, L. (1990). Exploring the architecture of the language-processing system. In G. Altmann (Ed.), Cognitive models of speech processing (pp. 409-433). Cambridge, MA: MIT Press.

Grosjean, F. (1980). Spoken word recognition processes and the gating paradigm. Perception \& Psychophysics, 28, 267-283.

GROSJEAN, F. (1988). Exploring the recognition of guest words in bilingual speech. Language \& Cognitive Processes, 3, 233-274.

Grosjean, F., Dommergues, J.-Y., Cornu, E., Guillelmon, D., \& BESSON, C. (1994). The gender-marking effect in spoken word recognition. Perception \& Psychophysics, 56, 590-598.

Huang, J. (1984). On the distribution and reference of empty pronouns. Linguistic Inquiry, 15, 531-574.

Li, C., \& Thompson, S. (1981). Mandarin Chinese: A functional reference grammar. Berkeley: University of California Press. 
LI, P. (1994). Understanding the time course of sentence comprehension A sentence gating study in Mandarin Chinese. In H.-W. Chang, J. T. Huang, C.-W. Hue, \& O. Tzeng (Eds.), Advances in the study of Chinese language processing (Vol. 1, pp. 303-323). Taipei: National Taiwan University Press.

LI, P. (in press). Spoken word recognition of code-switched words by Chinese-English bilinguals. Journal of Memory \& Language.

Li, P., Bates, E., \& MacWhinney, B. (1993). Processing a language without inflections: A reaction time study of sentence interpretation in Chinese. Journal of Memory \& Language, 32, 169-192.

LiU, H., Bates, E., \& Li, P. (1992a). Grammaticality judgment in Chinese-English bilinguals: A gating experiment. In Proceedings of the 14th Annual Conference of the Cognitive Science Society (pp. 9599). Hillsdale, NJ: Erlbaum.

LiU, H., BATES, E., \& Li, P. (1992b). Sentence interpretation in bilingual speakers of English and Chinese. Applied Psycholinguistics, 13, 451-484

LiU, H., Bates, E., Powell, T., \& Wulfeck, B. (in press). Single-word shadowing and the study of lexical access. Applied Psycholinguistics.

LU, J.-M. (1980). Hanyu kouyu jufa-li -de yiwei xianxiang [On reversed sentence patterns in spoken Chinese]. Zhongguo Yuwen, 1, 28-41.

MacDonald, M., Pearlmutter, N., \& Seidenberg, M. (1994). The lexical nature of syntactic ambiguity resolution. Psychological Review, 101, 676-703.

MacWhinney, B., \& Bates, E. (Eds.) (1989). The crosslinguistic study of sentence processing. New York: Cambridge University Press.

MARSLEN-WILSON, W. (1985). Speech shadowing and speech comprehension. Speech Comprehension, 4, 55-73.

MARSLEN-WILSON, W. (1987). Functional parallelism in spoken wordrecognition. Cognition, 25, 71-102.

MarsLEN-Wilson, W. (1990). Activation, competition, and frequency in lexical access. In G. Altmann (Ed.), Cognitive models of speech processing (pp. 148-172). Cambridge, MA: MIT Press.

McClelland, J., Rumelhart, D., \& The PDP Research Group (1986). Parallel distributed processing: Explorations in the microstructure of cognition (Vol. 2, pp. 58-121). Cambridge, MA: MIT Press.

MiaO, X.-C. (1981). Word order and semantic strategies in Chinese sentence comprehension. International Journal of Psycholinguistics, 8, 109-122.

MiaO, X.-C., Chen, G., \& YING, H. (1986). Sentence comprehension in Chinese. In M. Zhu (Ed.), Studies in child language development (pp. 40-53). Shanghai: East China Normal University Press.

SlowIACZEK, L. (1994). Semantic priming in a single-word shadowing task. American Journal of Psychology, 107, 245-260.

Sun, C.-F. (1991, May). Transitivity and the ba construction. Paper presented at the third North American Conference on Chinese Linguistics, Cornell University, Ithaca.

Tyler, L., \& Wessels, J. (1985). Is gating an on-line task? Evidence from naming latency data. Perception \& Psychophysics, 38, 217-222.

von Berger, E., Wulfeck, B., Bates, E., \& Fink, N. (in press). Developmental changes in real-time sentence processing. First Language.

ZHU, D.-X. (1982). Yufa jiangyi [Lectures on grammar]. Beijing: Commercial Press.

ZHU, D.-X. (1985). Yufa dawen [A dialogue on grammar]. Beijing: Commercial Press.

\section{NOTES}

1. Several standard grammatical abbreviations are used in this paper: SVO (subject-verb-object), SOV (subject-object-verb), OSV (objectsubject-verb), NVN (noun-verb-noun), NNV (noun-noun-verb), VNN (verb-noun-noun).

2. The grammatical judgments were based on two sources: (1) published, well-known linguistic analyses of spoken Chinese grammar, such as Chao (1968), C. Li and Thompson (1981), and Zhu (1982); (2) my own linguistic analyses, mainly for the incomplete sentence gates, since there have been no studies to my knowledge that have systematically investigated the grammaticality of these fragments in Chinese. The criterion for testing the grammaticality of these fragments is to see if they can be used alone in answering questions.

3. All remaining post hoc tests were conducted using Tukey's HSD measure. An alpha level of .05 was used for all post hoc tests for significance, and thus no $p$ values will be reported in the following.

4. A post hoc analysis revealed that other factors, for example, the degree of "animateness" of the noun phrases, might have contributed to the high proportion of first-noun choices in N $b a \mathrm{NV}$ sentences. Listeners (as indicated in their self-reports after the experiment) tended to choose stronger animals (e.g., elephant) as the agent rather than the weaker ones (e.g., cat) even though both are animate. This suggests that animacy is not a simple contrast between animate versus inanimate; rather, it may vary by degree along a continuum of animacy-inanimacy, as indicated by some typological linguistic studies (Comrie, 1989). Note that the assignment of a stronger or a weaker animal to the first or to the second noun was done randomly by the computer program, but by chance the assignment may not always be balanced.

An alternative explanation is that the verbs used in the test sentences all indicate aggressive meaning, thus motivating listeners' bias to take stronger animals as agents. I am grateful to an anonymous reviewer for this suggestion.

5 . These analyses may be obtained from the author upon request.

6. The absence of a word order effect might be due to the overwhelming strength that the bei marker has over word order in Chinese sentence comprehension. P. Li et al. (1993) indicated that bei wins over word order in case of competition.

7. A simultaneous interpreter anecdotally reported that during simultaneous interpretation, the interpreter has to start the translation of the sentence well before its end, and at the onset of the translation the interpreter has roughly predicted the contents of the whole sentence.

\section{APPENDIX A \\ Nouns and Verbs Used in the Test Sentences}

\section{Nouns}

banma, "zebra"; daxiang, "elephant"; daishu, "kangaroo"; gongji, "cock"; gouxiong, "bear"; haitun, "dolphin"; houzi, "monkey"; hudie, "butterfly"; jinyu, "goldfish"; laohu, "tiger"; laoshu, "mouse"; mianyang, "sheep"; qi'e, "penguin"; xiaogou, "dog"; xiaoma, "horse"; xiaomao, "cat"; xiaoniao, "bird"; xiaoniu, "cow"; xiaotu, "rabbit"; xiaoya, "duckling"; xiaozhu, "pig"; wugui, "turtle."

\section{Verbs}

chi-diao, "eat-up"; da-bai, "hit-defeat"; fang-zou, "release-go"; gan-pao, "drive-go"; ti-dao, "kick-down"; tui-kai, "push-open"; yao-zhu, "bite-hold"; zhua-zhu, "seize-hold"; zhuang-dao, "bump-fall."

\section{APPENDIX B}

Grammaticality for All Sentence Gates Used in the Experiments

\begin{tabular}{|c|c|c|c|c|}
\hline \multirow{2}{*}{$\begin{array}{c}\text { Type } \\
\text { Simple }\end{array}$} & \multicolumn{4}{|c|}{ Sentence Gates } \\
\hline & $\mathrm{N}$ & NV & NVN & \\
\hline & $\mathrm{N}$ & $\mathrm{NN}$ & NNV & \\
\hline & $\mathrm{V}$ & $\mathrm{VN}$ & $\mathrm{VNN}$ & \\
\hline \multirow[t]{9}{*}{$B a$} & ${ }^{*} b a$ & $b a N$ & $b a \mathrm{NN}$ & $b a \mathrm{NNV}$ \\
\hline & $N$ & $* N b a$ & $\mathrm{~N} b a \mathrm{~N}$ & NbaNV \\
\hline & $\mathrm{N}$ & NN & ${ }^{*} \mathrm{NN} b a$ & ${ }^{*} \mathrm{NN} b a \mathrm{~V}$ \\
\hline & ${ }^{*} b a$ & $b a N$ & $b a \mathrm{NV}$ & $* b a \mathrm{NVN}$ \\
\hline & $\mathrm{N}$ & *Nba & ${ }^{*} \mathrm{~N} b a \mathrm{~V}$ & ${ }^{*} \mathrm{~N} b a \mathrm{VN}$ \\
\hline & $\mathrm{N}$ & NV & ${ }^{*} \mathrm{NV} b a$ & ${ }^{*} \mathrm{NV} b a \mathrm{~N}$ \\
\hline & ${ }^{*} b a$ & $* b a \mathrm{~V}$ & ${ }^{*} b a \mathrm{VN}$ & ${ }^{*} b a \mathrm{VNN}$ \\
\hline & V & $* V b a$ & $* \mathrm{~V} b a \mathrm{~N}$ & *VbaNN \\
\hline & $\mathrm{V}$ & $\mathrm{VN}$ & *VN $b a$ & *VN $b a \mathrm{~N}$ \\
\hline \multirow[t]{9}{*}{$B e i$} & ${ }^{*} b e i$ & bei $\mathrm{N}$ & $b e i \mathrm{NN}$ & beiNNV \\
\hline & $\mathrm{N}$ & ${ }^{*} \mathrm{~N} b e i$ & $\mathrm{~N} b e i \mathrm{~N}$ & NbeiNV \\
\hline & $\mathrm{N}$ & $\mathrm{NN}$ & ${ }^{*} \mathrm{NN} b e i$ & NNbeiV \\
\hline & ${ }^{*} b e i$ & bei $\mathrm{N}$ & $b e i \mathrm{NV}$ & bei NVN \\
\hline & $\mathrm{N}$ & *Nbei & $\mathrm{Nbei \textrm {V }}$ & *N $b e i \mathrm{VN}$ \\
\hline & $\mathrm{N}$ & NV & *NVbei & NVbei $\mathrm{N}$ \\
\hline & *bei & beiV & ${ }^{*} b e i \mathrm{VN}$ & ${ }^{*} b e i \mathrm{VNN}$ \\
\hline & $\mathrm{V}$ & *Vbei & *Vbei $\mathrm{N}$ & ${ }^{*} \mathrm{~V} b e i \mathrm{NN}$ \\
\hline & V & $\mathrm{VN}$ & *VNbei & $\mathrm{VN}$ bei $\mathrm{N}$ \\
\hline
\end{tabular}

*Ungrammatical. 


\begin{tabular}{|c|c|c|c|c|c|c|c|c|c|c|c|}
\hline \multicolumn{12}{|c|}{ 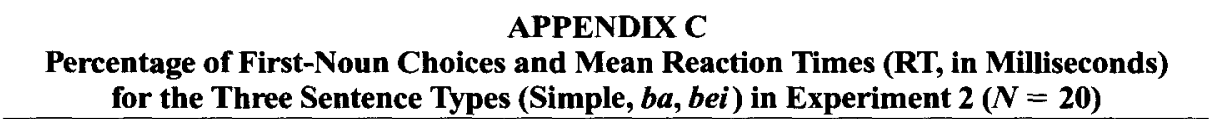 } \\
\hline & \multicolumn{3}{|c|}{ Simple } & \multicolumn{4}{|c|}{$b a$} & \multicolumn{4}{|c|}{ bei } \\
\hline & 1 & 2 & 3 & 1 & 2 & 3 & 4 & 1 & 2 & 3 & 4 \\
\hline \multicolumn{12}{|c|}{ First-Noun Choices for Gates $1-4$} \\
\hline$(*) \mathrm{NNV}$ & 66 & 56 & 50 & 66 & 28 & 44 & 45 & 45 & 71 & 64 & 59 \\
\hline (*)NVN & 74 & 89 & 88 & 51 & 28 & 16 & 18 & 67 & 79 & 86 & 78 \\
\hline (*)VNN & 52 & 17 & 23 & 31 & 35 & 31 & 33 & 41 & 41 & 44 & 31 \\
\hline $\mathrm{N}\left({ }^{*}\right) \mathrm{NV}$ & & & & 81 & 87 & 91 & 93 & 67 & 17 & 13 & 16 \\
\hline $\mathrm{N}\left({ }^{*}\right) \mathrm{VN}$ & & & & 46 & 75 & 72 & 66 & 56 & 14 & 13 & 19 \\
\hline $\mathrm{V}\left({ }^{*}\right) \mathrm{NN}$ & & & & 50 & 49 & 20 & 28 & 46 & 48 & 68 & 75 \\
\hline$N N(*) \mathrm{V}$ & & & & 63 & 53 & 51 & 49 & 72 & 60 & 59 & 48 \\
\hline$N V\left(^{*}\right) \mathrm{N}$ & & & & 55 & 79 & 84 & 78 & 80 & 91 & 70 & 57 \\
\hline $\mathrm{VN}\left({ }^{*}\right) \mathrm{N}$ & & & & 49 & 13 & 23 & 30 & 35 & 08 & 09 & 13 \\
\hline \multicolumn{12}{|c|}{ Mean RT for Gates $1-4$} \\
\hline$\left(^{*}\right) \mathrm{NNV}$ & 1,465 & 1,798 & 2,218 & 1,382 & 1,551 & 1,930 & 2,193 & 1,321 & 1,578 & 1,841 & 2,022 \\
\hline$(*) \mathrm{NVN}$ & 1,487 & 1,554 & 1,669 & 1,262 & 1,461 & 1,502 & 1,607 & 1,454 & 1,525 & 1,671 & 1,886 \\
\hline (*)VNN & 1,561 & 1,680 & 1,937 & 1,260 & 1,673 & 2,116 & 2,307 & 1,487 & 1,675 & 1,937 & 2,272 \\
\hline $\mathrm{N}\left({ }^{*}\right) \mathrm{NV}$ & & & & 1,320 & 1,433 & 1,452 & 1,620 & 1,490 & 1,590 & 1,696 & 1,782 \\
\hline $\mathrm{N}\left({ }^{*}\right) \mathrm{VN}$ & & & & 1,446 & 1,388 & 1,699 & 2,078 & 1,437 & 1,574 & 1,691 & 1,746 \\
\hline $\mathrm{V}\left({ }^{*}\right) \mathrm{NN}$ & & & & 1,484 & 1,720 & 1,826 & 2,158 & 1,527 & 1,664 & 1,767 & 1,968 \\
\hline $\mathrm{NN}\left({ }^{*}\right) \mathrm{V}$ & & & & 1,366 & 1,742 & 1,970 & 2,226 & 1,477 & 1,852 & 2,092 & 2,200 \\
\hline $\mathrm{NV}\left({ }^{*}\right) \mathrm{N}$ & & & & 1,514 & 1,571 & 1,714 & 2,052 & 1,392 & 1,547 & 1,791 & 1,837 \\
\hline $\mathrm{VN}\left({ }^{*}\right) \mathrm{N}$ & & & & 1,534 & 1,544 & 1,815 & 2,022 & 1,441 & 1,565 & 1,739 & 1,950 \\
\hline
\end{tabular}

Note- $\left(^{*}\right)$ indicates the position where the $b a$ or the bei marker occurs; this does not apply to simple sentences.

\begin{tabular}{|c|c|c|c|}
\hline \multicolumn{4}{|c|}{$\begin{array}{l}\text { APPENDIX D } \\
\text { Percentage of First-Noun Choices and Mean Reaction } \\
\text { Times (RT, in Milliseconds) for the Three Sentence } \\
\text { Types (Simple, ba, bei) in Experiment } 3(N=20)\end{array}$} \\
\hline & Simple & $B a$ & $B e i$ \\
\hline \multicolumn{4}{|c|}{ First-Noun Choices } \\
\hline$\left.{ }^{*}\right) \mathrm{NNV}$ & 50 & 53 & 54 \\
\hline$\left({ }^{*}\right) \mathrm{NVN}$ & 91 & 20 & 65 \\
\hline$\left({ }^{*}\right) \mathrm{VNN}$ & 19 & 26 & 31 \\
\hline $\mathrm{N}\left({ }^{*}\right) \mathrm{NV}$ & & 98 & 18 \\
\hline $\mathrm{N}\left({ }^{*}\right) \mathrm{VN}$ & & 51 & 23 \\
\hline $\mathrm{V}\left({ }^{*}\right) \mathrm{NN}$ & & 26 & 70 \\
\hline $\mathrm{NN}\left({ }^{*}\right) \mathrm{V}$ & & 56 & 58 \\
\hline $\mathrm{NV}\left({ }^{*}\right) \mathrm{N}$ & & 74 & 34 \\
\hline $\mathrm{VN}\left({ }^{*}\right) \mathrm{N}$ & & 43 & 11 \\
\hline \multicolumn{4}{|c|}{ Mean RTs } \\
\hline$\left.{ }^{*}\right) \mathrm{NNV}$ & 2,618 & 2,708 & 2,589 \\
\hline$\left.{ }^{*}\right) \mathrm{NVN}$ & 2,203 & 2,301 & 2,498 \\
\hline (*)VNN & 2,345 & 2,745 & 2,602 \\
\hline $\mathrm{N}(*) \mathrm{NV}$ & & 2,235 & 2,538 \\
\hline $\mathrm{N}(*) \mathrm{VN}$ & & 2,615 & 2,547 \\
\hline $\mathrm{V}(*) \mathrm{NN}$ & & 2,564 & 2,641 \\
\hline$N N(*) V$ & & 2,696 & 2,810 \\
\hline$N V\left(^{*}\right) \mathrm{N}$ & & 2,668 & 2,664 \\
\hline $\mathrm{VN}\left({ }^{*}\right) \mathrm{N}$ & & 2,754 & 2,527 \\
\hline
\end{tabular}

Note- $\left({ }^{*}\right)$ indicates the position where the $b a$ or the bei marker occurs; this does not apply to simple sentences.

(Manuscript received November 11, 1994; revision accepted for publication August 21, 1995.) 